\title{
The Area under the Curve (AUC) of Oral Glucose Tolerance Test (OGTT) Could Be a Measure Method of Hyperglycemia in All Pregnant Women
}

\author{
Congyue Zhang, Yumei Wei, Weijie Sun, Huixia Yang \\ Department of Obstetrics and Gynecology, Peking University First Hospital, Beijing, China \\ Email: sophie1166@163.com
}

How to cite this paper: Zhang, C.Y., Wei, Y.M., Sun, W.J. and Yang, H.X. (2019) The Area under the Curve (AUC) of Oral Glucose Tolerance Test (OGTT) Could Be a Measure Method of Hyperglycemia in All Pregnant Women. Open Journal of Obstetrics and Gynecology, 9, 186-195.

https://doi.org/10.4236/ojog.2019.92019

Received: January 13, 2019

Accepted: February 8, 2019

Published: February 11, 2019

Copyright $\odot 2019$ by author(s) and Scientific Research Publishing Inc. This work is licensed under the Creative Commons Attribution International License (CC BY 4.0).

http://creativecommons.org/licenses/by/4.0/

\begin{abstract}
Objective: We adopted the area under the curve (AUC) of oral glucose tolerance test (OGTT) as a measure method of the severity of maternal hyperglycemia and investigated its relationship with adverse perinatal outcomes among women with and without gestational diabetes mellitus (GDM). Research design and methods: This is a retrospective cohort study. Our study group collected the medical records of 15,296 women who received perinatal care in 15 hospitals in Beijing and who delivered from July 1, 2013, to December 31, 2013. And several original articles on this cohort have been published. In this study, we analyze the relationship between AUC and adverse perinatal outcomes, so that in multiple pregnant cases, patients with pre-pregnancy diabetes, hypertension, and abnormal kidney function and those who did not receive a 75-g OGTT were excluded. A Chi-squared test and logistic regression analysis were used to determine the associations. Results: In total, 13,561 women were included. As the AUC of OGTT increased, the prevalence of macrosomia (odds ratio [OR] 1.059, 95\% confidence interval [95\% CI] 1.029 $1.090, \mathrm{p}<0.001$ ) and hypertensive diseases (OR 1.106, 95\% CI $1.064-1.149, \mathrm{p}$ $<0.001$ ) also increased. For patients with same levels of AUC values, no significant differences in the risk of macrosomia, preterm birth and neonatal complications were observed between the GDM and non-GDM groups. Women with an AUC higher than $14.20\left(\mathrm{mmol}^{*} \mathrm{~h} / \mathrm{L}\right)$ had a higher risk of adverse outcomes regardless of the presence of GDM. Conclusions: The AUC could be a measure method of the severity of maternal hyperglycemia, and women with a high AUC should undergo aggressive management to avoid adverse outcomes regardless of the presence of GDM.
\end{abstract}




\section{Keywords}

AUC, GDM, OGTT, Measure Method, Hyperglycemia

\section{Introduction}

The prevalence of gestational diabetes mellitus (GDM) is increasing worldwide. Recent estimates have suggested that approximately $17.5 \%$ of pregnant women suffer from GDM throughout China [1]. Our previous study showed that in this cohort, 2987 (19.7\%) women had GDM and 208 (1.4\%) had diabetes in pregnancy (DIP) as a result of high prepregnancy body mass index (BMI) and BMI gain before 24 weeks [2]. We have proved that hyperglycemia lead to adverse perinatal outcomes, including high cesarean section rate [2] [3] [4]. The Hyperglycemia and Adverse Pregnancy Outcome (HAPO) study demonstrated that the risk of adverse perinatal outcomes continuously increases as a result of maternal hyperglycemia, even for patients within ranges previously considered normal for pregnancy [5]. However, the International Association of Diabetes and Pregnancy Study Group (IADPSG) adopted the threshold value of an odds ratio (OR) of 1.75 relative to the mean for adverse pregnancy outcomes. Using an oral glucose tolerance test (OGTT) as the diagnostic criterion, women with one glucose value above the criterion were considered to have GDM [6]. Our team has found that various characteristics of OGTTs are associated with different adverse outcomes. Fasting hyperglycemia had clear association with macrosomia and cesarean section [7]. To combine the three values of OGTT, we adopted the area under the curve (AUC) of OGTT, which is the geometric mean value, to assess the severity of maternal hyperglycemia and to evaluate the risk of adverse perinatal outcomes. As shown in the curve in Figure 1, the AUC of the time-blood glucose curve of the OGTT approximately equals the areas of two trapezoids as follows: $(0 \mathrm{~h}$ blood glucose $+1 \mathrm{~h}$ blood glucose $) \times 1 / 2+(1 \mathrm{~h}$ blood glucose $+2 \mathrm{~h}$ blood glucose $) \times 1 / 2$, which equals the following: $1 \mathrm{~h}$ blood glucose $+(0 \mathrm{~h}$ blood glucose $+2 \mathrm{~h}$ blood glucose)/2. Using this formula, we can easily calculate the

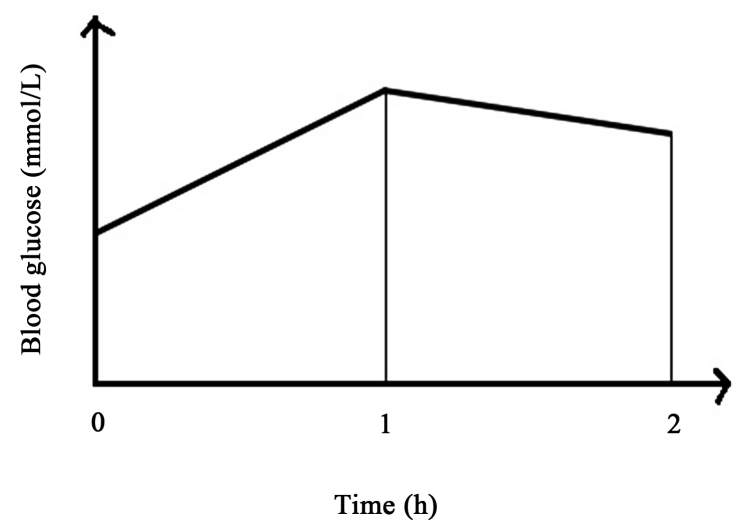

Figure 1. AUC of the time-blood glucose curve of OGTT. 
AUC of OGTT. In another research, we have proved that in women with gestational diabetes, higher AUC implied higher risk of adverse perinatal outcomes [8]. In this study, we wanted to make sure if AUC could be a measure method of hyperglycemia in all pregnant women no matter if she had GDM or not.

\section{Methods}

\subsection{Ethical Approval}

This study was approved by the Ethics Committee of Peking University First Hospital (No. 2013 [578]). All participants provided written informed consent, and the Ethics Committee approved the consent procedure.

\subsection{Study Population and Definitions}

We conducted a retrospective cohort study of medical records from the "Systemic Random Sampling Survey on the Prevalence of Gestational Diabetes in Beijing (GPS)". A total of 15,296 women who received perinatal care in 15 hospitals in Beijing delivered between July 1, 2013, and December 31, 2013. Of these, 190 were multiple pregnant. Among the single preganant women, 237 were diagnosed with diabetes mellitus or hypertension before pregnancy, 13 had abnormal kidney function and 1295 did not undergo an OGTT at 24 - 28 weeks. Finally, 13,561 women were included.

Women were diagnosed with diabetes pre-pregnancy diabetes or GDM according to the Chinese Diagnostic criteria for gestational diabetes mellitus (WS 331-2011) [9]. Gestational hypertensive disease refers to gestational hypertension, preeclampsia and eclampsia according to the guidelines of Hypertension in Pregnancy from the American College of Obstetrics and Gynecology (ACOG), 2013 [10]. Macrosomia was defined as a birth weight of more than $4000 \mathrm{~g}$, while preterm birth was defined as a delivery at a gestational age $<37$ weeks and $\geq 28$ weeks. Neonatal complications included neonatal hypoglycemia (glucose values $<35 \mathrm{mg} / \mathrm{dL}$ by heel stick within $2 \mathrm{~h}$ of birth and before the first breastfeeding), neonatal hyperbilirubinemia (values $>$ 95th percentile), asphyxia neonatorum (Apgar score $\leq 7$ ) and infants hospitalized in the pediatric unit for other reasons within 7 days [11]. There were only no case of perinatal death not result of preterm birth and therapeutic fatal termination. So perinatal death was not included in neonatal complications.

The variable in this study was AUC of OGTT. We divided the cohort into subgroups according to AUC value and analyzed the prevalence of adverse perinatal outcomes in different groups.

\subsection{Statistical Analysis}

Statistical analysis was conducted with SPSS version 20.0 (IBM, Chicago, IL, USA) software. T tests, Pearson's Chi-squared or Fisher's exact tests and logistic regression tests were performed. The measurement data are presented as the mean \pm standard deviation $(\mathrm{SD})$. Incidence data are presented as the number 
and frequency (n [\%]). ORs and the $95 \%$ confidence interval (95\% CI) are also presented. A value of $\mathrm{p}<0.05$ was considered statistically significant.

\section{Results}

In total, $2547(18.8 \%)$ women had GDM. General details of the cohort are shown in Table 1.

The mean \pm SD values of the AUC $\left(\mathrm{mmol}^{*} \mathrm{~h} / \mathrm{L}\right)$ were $12.50 \pm 1.70,15.96 \pm$ 2.28 and $13.19 \pm 2.26$ in the non-GDM, GDM and total group, respectively. The AUC of the diagnostic criteria was 16.80; therefore, those with an AUC $\geq 16.80$ were considered to have GDM. The data were divided into 6 groups based on the AUC: $<10.80$ (mean-SD in the non-GDM group), $10.80-12.49$ (mean in the non-GDM group), 12.50 - 14.19 (mean \pm SD in the non-GDM group), 14.20 15.99 (mean in the GDM group), $16.00-16.79$ (AUC of the diagnostic criteria) and $\geq 16.80$.

Groups 1 and 2 rarely exhibited GDM, while most of Group 5 had GDM. We analyzed the prevalence of adverse outcomes in different AUC groups relative to the total non-GDM group. Group 1 had a lower risk of the four analyzed adverse outcomes, among which the prevalence rates of macrosomia and hypertensive diseases were significant. Groups 2 and 3 had a similar prevalence of adverse outcomes as the non-GDM group. Group 4 had a significantly higher risk of adverse outcomes. Groups 5 and 6 also had a higher risk of adverse outcomes, which was statistically significant (Table 2).

Table 1. General details of the cohort.

\begin{tabular}{ccc}
\hline Parameters & $\begin{array}{c}\text { Non-GDM } \\
(\mathrm{n}=11,009)\end{array}$ & $\begin{array}{c}\text { GDM } \\
(\mathrm{n}=2552)\end{array}$ \\
\hline Maternal delivery age $(\mathrm{y})$ & $28.04 \pm 4.09$ & $29.73 \pm 4.40^{*}$ \\
BMI pre-pregnancy $\left(\mathrm{kg} / \mathrm{m}^{2}\right)$ & $21.32 \pm 3.35$ & $22.55 \pm 3.86^{*}$ \\
1st trimester FPG $(\mathrm{mmol} / \mathrm{L})$ & $4.66 \pm 0.41$ & $4.96 \pm 0.46^{*}$ \\
Family history $(\mathrm{n}[\%])$ & $1367(12.4)$ & $549(21.5)^{*}$ \\
\hline
\end{tabular}

${ }^{*} \mathrm{p}<0.05$ versus the non-GDM group.

Table 2. Adverse perinatal outcomes in different AUC groups.

\begin{tabular}{|c|c|c|c|c|c|c|c|}
\hline \multirow[b]{2}{*}{ Group } & \multirow[b]{2}{*}{ AUC } & \multirow[b]{2}{*}{$\mathrm{n}$} & \multirow{2}{*}{$\begin{array}{l}\text { GDM } \\
\mathrm{n}(\%)\end{array}$} & \multicolumn{4}{|c|}{ Adverse Outcomes n (\%) } \\
\hline & & & & Macrosomia & $\begin{array}{l}\text { Preterm } \\
\text { birth }\end{array}$ & $\begin{array}{c}\text { Hypertensive } \\
\text { diseases }\end{array}$ & $\begin{array}{c}\text { Neonatal } \\
\text { complications }\end{array}$ \\
\hline 1 & $<10.80$ & 1775 & $23(1.29)$ & $92(5.18)^{*}$ & $56(3.15)$ & $33(1.86)^{*}$ & $176(9.92)$ \\
\hline 2 & $10.80-12.49$ & 3741 & $130(3.28)$ & $252(6.74)$ & $142(3.80)$ & $127(3.39)$ & $396(10.6)$ \\
\hline 3 & $12.50-14.19$ & 3992 & 367 (9.19) & $326(8.17)$ & $185(4.63)$ & $136(3.41)$ & $472(11.8)$ \\
\hline 4 & $14.20-15.99$ & 2630 & $695(26.4)$ & $247(9.39)^{\star}$ & $131(4.98)^{\star}$ & $132(5.02)^{\star}$ & $331(12.6)^{\star}$ \\
\hline 5 & $16.00-16.79$ & 598 & $512(85.6)$ & $61(10.2)^{*}$ & $29(4.85)$ & $31(5.18)^{*}$ & $75(12.6)$ \\
\hline 6 & $\geq 16.80$ & 825 & $825(100)$ & $72(8.72)$ & $60(7.27)^{\star}$ & $60(7.27)^{*}$ & $125(15.2)^{\star}$ \\
\hline Non-GDM & & 11,009 & & $819(7.44)$ & $452(4.10)$ & $361(3.28)$ & $1229(11.2)$ \\
\hline
\end{tabular}

${ }^{*} \mathrm{p}<0.05$ versus the non-GDM group. 
Because patients rarely had GDM in Groups 1 and 2, and most had GDM in Groups 5 and 6, we compared the prevalence of adverse outcomes in patients with and without GDM in Groups 3 and 4. As is shown in Table 3, within the same group, the prevalence rates of macrosomia, preterm birth and neonatal complications were not significantly different between the non-GDM and GDM groups. However, in Group 4, patients with GDM had a higher risk of hypertensive diseases.

Among the 13,561 women included in this cohort, 11,892 had complete data of risk factors such as delivery age, height, body weight before pregnancy and before delivery, $1^{\text {st }}$ trimester fasting plasma glucose (FPG), family history and OGTT results. We analyzed suspected risk factors by single factor logistic regression. Body mass index (BMI, kg/m²) pre-pregnancy (OR 1.148, 95\% CI 1.126 - 1.170, p < 0.001) (OR 1.215, 95\% CI 1.185 - 1.247, p < 0.001), BMI increment during pregnancy (OR 1.224, 95\% CI $1.192-1.257, \mathrm{p}<0.001)$ (OR 1.223, 95\% CI $1.179-1.269, \mathrm{p}<0.001)$ and AUC (OR 1.082, 95\% CI $1.043-1.124, \mathrm{p}<0.001$ ) (OR 1.073, 95\% CI $1.019-1.129, \mathrm{p}=0.007)$ influenced the prevalence of macrosomia as well as hypertensive diseases. But there was no significant influence on the prevalence of preterm birth and neonatal complications. While other risk factors were not significantly related to adverse outcomes. A multiple Logistic regression was performed to analyze the risk factors above (Table 4). We also drew receiver operating characteristic (ROC) curves of AUC for macrosomia and hypertensive diseases. However, the AUCs of ROC curves were 0.554 (95\% CI $0.535-0.573, \mathrm{p}=0.009)$ for macrosomia and 0.591 (95\% CI $0.564-0.618, \mathrm{p}=$ 0.014 ) for hypertensive diseases. And there was no perfect cut-off value.

\section{Discussion}

In 2011, the National Health and Family Planning Commission of China adopted testing and diagnostic criteria based on the IADPSG guidelines, according to

Table 3. Adverse outcomes in Groups 3 and 4 for patients with and without GDM.

\begin{tabular}{|c|c|c|c|c|c|c|c|}
\hline & \multirow[b]{2}{*}{ Group } & \multirow[b]{2}{*}{$\mathrm{n}$} & & \multicolumn{4}{|c|}{ Adverse outcomes } \\
\hline & & & & Macrosomia & $\begin{array}{l}\text { Preterm } \\
\text { birth }\end{array}$ & $\begin{array}{c}\text { Hypertensive } \\
\text { diseases }\end{array}$ & $\begin{array}{c}\text { Neonatal } \\
\text { complications }\end{array}$ \\
\hline \multirow{2}{*}{3} & GDM & 367 & & $31(8.45)$ & $23(6.27)$ & $14(3.81)$ & $43(11.7)$ \\
\hline & Non-GDM & 3625 & & $295(8.14)$ & $162(4.47)$ & $122(3.36)$ & $429(11.8)$ \\
\hline \multirow{6}{*}{4} & & & OR & 1.003 & 1.019 & 1.005 & 0.999 \\
\hline & & & $95 \% \mathrm{CI}$ & $0.971-1.037$ & $0.992-1.047$ & $0.984-1.026$ & $0.960-1.039$ \\
\hline & GDM & 695 & & $66(9.50)$ & $31(4.46)$ & $49(7.05)^{*}$ & $95(13.7)$ \\
\hline & Non-GDM & 1935 & & $181(9.35)$ & $100(5.17)$ & $83(4.29)$ & $235(12.1)$ \\
\hline & & & OR & 1.002 & 0.993 & 1.036 & 1.018 \\
\hline & & & $95 \% \mathrm{CI}$ & $0.974-1.030$ & $0.974-1.012$ & $1.011-1.061$ & $0.984-1.053$ \\
\hline
\end{tabular}

Data are presented as $\mathrm{n}(\%) .{ }^{\star} \mathrm{p}<0.05$ versus the non-GDM group. 
Table 4. Multiple logistic regression of risk factors.

\begin{tabular}{|c|c|c|c|c|}
\hline \multirow{2}{*}{\multicolumn{2}{|c|}{ Adverse outcomes }} & \multicolumn{3}{|c|}{ Risk factors } \\
\hline & & BMI pre-pregnancy & BMI increment & AUC \\
\hline \multirow{4}{*}{ Macrosomia } & OR & 1.148 & 1.225 & 1.059 \\
\hline & $95 \% \mathrm{CI}$ & $1.126-1.170$ & $1.193-1.257$ & $1.029-1.090$ \\
\hline & $\mathrm{p}$ & $<0.001$ & $<0.001$ & $<0.001$ \\
\hline & Percentage correct & & $92.2 \%$ & \\
\hline \multirow{4}{*}{$\begin{array}{l}\text { Hypertensive } \\
\text { diseases }\end{array}$} & OR & 1.217 & 1.219 & 1.106 \\
\hline & $95 \% \mathrm{CI}$ & $1.186-1.249$ & $1.175-1.264$ & $1.064-1.149$ \\
\hline & $\mathrm{p}$ & $<0.001$ & $<0.001$ & $<0.001$ \\
\hline & Percentage correct & & $96.3 \%$ & \\
\hline
\end{tabular}

which more pregnant women were classified as having GDM. Moreover, these GDM patients had different hyperglycemia characteristics, which may lead to different pregnancy outcomes. Researchers have searched for indicators to assess the severity of GDM, such as hemoglobin Alc (HbAlc) and glycated albumin (GA) levels [12] [13]. Previous study from our research group showed that various characteristics of OGTTs are associated with different adverse outcomes. Fasting hyperglycemia was associated with macrosomia, large size for gestational age (LGA) and cesarean delivery. Hyperglycemia in the OGTT-2 $\mathrm{h}$ was more likely to lead to preterm birth [7]. However, they did not combine the three levels of blood glucose in the OGTT into a single assessment, which was performed in this study. In 2009, Korean researchers adopted the area under the curve of $100 \mathrm{~g}$ OGTT as a quantitative method of GDM and found that the incidence of LGA increased with the AUC, which was quite similar in our study [14]. However, their calculating formula was complicated and hard to extend in clinical use.

Hyperglycemia contributes to many adverse maternal, fetal and neonatal outcomes, such as macrosomia, LGA, small for gestational age (SGA), preterm birth, gestational hypertension, cesarean delivery, neonatal hypoglycemia, neonatal hyperbilirubinemia, hyperinsulinemia and other long-term effects [5]. Because the characteristics of this cohort and the relationship between hyperglycemia and adverse outcomes, neonatal complications included, had been discussed in our previous studies [2] [3] [4] [7], we did not aim at the topics above in this study. For the purpose of statistical analysis. We combined normal neonatal complications.

We found consistent trends between the AUC and the frequencies of adverse outcomes, except that the prevalence of macrosomia declined in Group 6 (AUC $\geq 16.80$ ). This result might have occurred because all patients in this group had GDM, who got additional management, including diet, exercise and insulin therapy, which led to a decline in the prevalence of macrosomia. Additionally, severe hyperglycemia or overly strict control of blood glucose may result in in- 
fants that are SGA [15] [16]. In Groups 4 to 6, in which patients had an AUC $\geq$ 14.20 (mean \pm SD of the non-GDM group), the prevalence of adverse outcomes was significantly higher than in the normal group (except for Group 5, the lack of a significant difference might be a result of the small sample size). This result also conformed to a linear risk factor of the normal distribution.

No significant difference was observed in the prevalence of adverse outcomes between the GDM and non-GDM groups when the AUC was similar, except for hypertensive diseases in Group 4. It is clear that intervention greatly contributed to this result [17] [18]. For ethical reasons, we cannot determine whether the results would be similar for a group without intervention or a control GDM group. A previous meta-analysis that included 10 studies involving 3881 patients indicated that GDM treatment significantly reduced the risk of macrosomia (RR, 0.47 ; 95\% CI, 0.38 - 0.57) and gestational hypertension (RR, 0.68; 95\% CI, 0.53 0.87 ) without causing any significant increase in the risk of SGA infants [19]. However, this study did not take the severity of GDM into account.

Metabolic abnormalities, such as increased circulating leptin, glucose, insulin, and lipids, are likely to increase the risk of preeclampsia in obese women. This is particularly relevant for obese pregnant women with GDM, for whom all of these factors are increased [20]. In Group 4, in which the AUC was $\geq 14.20$; the prevalence of hypertensive diseases was higher in the GDM group even though they were undergoing intervention. This result indicated that in patients with overt metabolic abnormalities, intervention may not reduce the risk of hypertensive diseases. Previous studies have shown similar comprehensive results. A cohort study including 2954 singleton pregnancies suggested that insulin resistance on an OGTT was associated with later development of gestational hypertension, but not preeclampsia, independent of maternal BMI [21]. Another study suggested that women who develop pregnancy-induced hypertension may be metabolically challenged at early stages of pregnancy with hyperinsulinism and insulin insensitivity [22]. It is accepted that obesity and other metabolic abnormalities make a difference in placental function and fetal development, which lead to several complications, such as GDM and hypertensive diseases [23].

The BMI pre-pregnancy and gestational weight gain (BMI increments) were significantly associated with macrosomia and hypertensive diseases, which has been suggested in many previous studies [2] [24] [25] [26] [27]. The AUC could be a risk factor and assessment indicator for pregnancy outcomes. GDM has been shown to contribute to adverse outcomes, and our results were obtained from patients who were diagnosed with GDM and were undergoing intervention and management. There were several limitations in this study. First, the influence of the intervention could not be quantified, and data were obtained from 15 hospitals, with different levels of effectiveness of the intervention. Additionally, the prevalence of adverse outcomes might be affected by many other risk factors, which were not included in this study. So far, deeper statistical analyse and long-term follow-up were carried on about this cohort. 


\section{Conclusion}

The AUC could be a measure method of the severity of maternal hyperglycemia. Women with a higher AUC had a higher risk of adverse pregnancy outcomes. Those with an AUC $\geq 14.20$ should undergo aggressive management regardless of the presence of GDM. For patients with similar AUC values, those with GDM who underwent effective intervention and management had similar pregnancy outcomes to non-GDM patients, except for hypertensive diseases.

\section{Conflicts of Interest}

The authors declare no conflicts of interest regarding the publication of this paper.

\section{References}

[1] Zhu, W.W., Yang, H.X., Wei, Y.M., Yan, J., Wang, Z.L., Li, X.L., et al. (2013) Evaluation of the Value of Fasting Plasma Glucose in the First Prenatal Visit to Diagnose Gestational Diabetes Mellitus in China. Diabetes Care, 36, 586-590.

https://doi.org/10.2337/dc12-1157

[2] Zhu, W.W., Yang, H.X., Wang, C., Su, R.N., Feng, H. and Kapur, A. (2017) High Prevalence of Gestational Diabetes in Beijing: Effect of Maternal Birth Weight and Other Risk Factors. Chinese Medical Journal, 130, 1019-1125. https://doi.org/10.4103/0366-6999.204930

[3] Song, G., Wei, Y.M., Zhu, W.W. and Yang, H.X. (2017) Cesarean Section Rate in Singleton Primiparae and Related Factors in Beijing, China. Chinese Medical Journal, 130, 2395-2401. https://doi.org/10.4103/0366-6999.216415

[4] Zhu, Y.C., Yang, H.X., Wei, Y.M., et al. (2017) Analysis of Correlation Factors and Pregnancy Outcomes of Hypertensive Disorders of Pregnancy-A Second Analysis of a Random Sampling in Beijing, China. The Journal of Maternal-Fetal \& Neonatal Medicine, 30, 751-754. https://doi.org/10.1080/14767058.2016.1186161

[5] HAPO Study Cooperative Research Group (2008) Hyperglycemia and Adverse Pregnancy Outcomes. The New England Journal of Medicine, 358, 1991-2002. https://doi.org/10.1056/NEJMoa0707943

[6] International Association of Diabetes Pregnancy Study Groups Consensus Panel (2010) International Association of Diabetes and Pregnancy Study Groups Recommendations on the Diagnosis and Classification of Hyperglycemia in Pregnancy. Diabetes Care, 33, 676-682. https://doi.org/10.2337/dc09-1848

[7] Feng, H., Zhu, W.W., Yang, H.X., Wei, Y.M., Wang, C., Su, R.N., et al. (2017) Relationship between Oral Glucose Tolerance Test Characteristics and Adverse Pregnancy Outcomes among Women with gestational Diabetes Mellitus. Chinese Medical Journal, 130, 1012-1018. https://doi.org/10.4103/0366-6999.204928

[8] Zhang, C.Y., Su, S.P. and Yang, H.X. (2015) Impact of Area under the Curve of Oral Glucose Tolerance Test on Pregnant Women with Gestational Diabetes Mellitus. Chinese Journal of Obstetrics and Gynecology, 50, 658-663.

[9] Yang, H.X. (2012) Diagnostic Criteria for Gestational Diabetes Mellitus (WS 331-2011). Chinese Medical Journal, 125, 1212-1213.

[10] American College of Obstetricians and Gynecologists (2013) Hypertension in Pregnancy. Obstetrics \& Gynecology, 122, 1122-1131. 
https://doi.org/10.1097/01.AOG.0000437382.03963.88

[11] Bhutani, V.K., Johnson, L. and Sivieri, E.M. (1999) Predictive Ability of a Predischarge Hour-Specific Serum Bilirubin for Subsequent Significant Hyperbilirubinemia in Healthy Term and Near-Term Newborns. Pediatrics, 103, 6-14. https://doi.org/10.1542/peds.103.1.6

[12] Rudland, V.L., Hinchcliffe, M., Pinner, J., Cole, S., Mercorella, B., Molyneaux, L., et al. (2016) Identifying Glucokinase Monogenic Diabetes in a Multiethnic Gestational Diabetes Mellitus Cohort: New Pregnancy Screening Criteria and Utility of HbA1c. Diabetes Care, 39, 50-52. https://doi.org/10.2337/dc15-1001

[13] Hashimoto, K. and Koga, M. (2015) Indicators of Glycemic Control in Patients with Gestational Diabetes Mellitus and Pregnant Women with Diabetes Mellitus. World Journal of Diabetes, 6, 1045-1056. https://doi.org/10.4239/wjd.v6.i8.1045

[14] Kim, S., Min, W.K., Chun, S., et al. (2009) Quantitative Risk Estimation for Large for Gestational Age Using the Area under the 100-g Oral Glucose Tolerance Test Curve. Journal of Clinical Laboratory Analysis, 23, 231-236. https://doi.org/10.1002/jcla.20326

[15] Goldstein, R.F., Abell, S.K., Ranasinha, S., Misso, M., Boyle, J.A., Black, M.H., et al. (2017) Association of Gestational Weight Gain with Maternal and Infant Outcomes: A Systematic Review and Meta-Analysis. JAMA, 317, 2207-2225. https://doi.org/10.1001/jama.2017.3635

[16] Harper, L.M., Tita, A. and Biggio, J.R. (2015) The Institute of Medicine Guidelines for Gestational Weight Gain after a Diagnosis of Gestational Diabetes and Pregnancy Outcomes. American Journal of Perinatology, 32, 239-246.

[17] Wei, Y.M., Yang, H.X., Zhu, W.W., Yang, H.Y., Li, H.X. and Kapur, A. (2015) Effects of Intervention to Mild GDM on Outcomes. The Journal of Maternal-Fetal \& Neonatal Medicine, 28, 928-931. https://doi.org/10.3109/14767058.2014.937697

[18] Carolan, O.M.C. (2016) Educational and Intervention Programmes for Gestational Diabetes Mellitus (GDM) Management: An Integrative Review. Collegian, 23, 103-114. https://doi.org/10.1016/j.colegn.2015.01.001

[19] Poolsup, N., Suksomboon, N. and Amin, M. (2014) Effect of Treatment of Gestational Diabetes Mellitus: A Systematic Review and Meta-Analysis. PLoS ONE, 9 , e92485.

[20] Spradley, F.T. (2017) Metabolic Abnormalities and Obesity's Impact on the Risk for Developing Preeclampsia. American Journal of Physiology-Regulatory Integrative and Comparative Physiology, 312, R5-R12.

[21] Kun, A. (2011) Insulin Resistance Is Associated with Gestational Hypertension and Not with Preeclampsia: A Population-Based Screening Study. Gynecologic and $O b-$ stetric Investigation, 71, 256-261. https://doi.org/10.1159/000320326

[22] Kayemba-Kay's, S., Peters, C., Geary, M.P., Hill, N.R., Mathews, D.R. and Hindmarsh, P.C. (2013) Maternal Hyperinsulinism and Glycaemic Status in the First Trimester of Pregnancy Are Associated with the Development of Pregnancy-Induced Hypertension and Gestational Diabetes. European Journal of Endocrinology, 168, 413-418. https://doi.org/10.1530/EJE-12-0609

[23] Howell, K.R. and Powell, T.L. (2017) Effects of Maternal Obesity on Placental Function and Fetal Development. Reproduction, 153, R97.

[24] Li, N., Liu, E., Guo, J., Pan, L., Li, B., Wang, P., et al. (2013) Maternal Pre-Pregnancy Body Mass Index and Gestational Weight Gain on Pregnancy Outcomes. PLoS ONE, 8, e82310. 
[25] Wei, Y.M., Yang, H.X., Zhu, W.W., Liu, X.Y., Meng, W.Y., Wang, Y.Q., et al. (2016) Risk of Adverse Pregnancy Outcomes Stratified for Pre-Pregnancy Body Mass Index. The Journal of Maternal-Fetal \& Neonatal Medicine, 29, 2205-2209.

[26] Billionnet, C., Mitanchez, D., Weill, A., et al. (2017) Gestational Diabetes and Adverse Perinatal Outcomes from 716,152 Births in France in 2012. Diabetologia, 60, 636. https://doi.org/10.1007/s00125-017-4206-6

[27] Catalano, P.M. and Shankar, K. (2017) Obesity and Pregnancy: Mechanisms of Short Term and Long Term Adverse Consequences for Mother and Child. BMJ, $356, \mathrm{j} 1$. 